long term and as part of a network, while respecting the child as a full participant and involving his or her parents. School health services, educational teams and health professionals must have the back up of legislation and political involvement in support of the prevention projects established. Schools and the PSE must contribute to the valorisation of self-worth and the awakening of critical thinking without which changes of behaviour will fail to materialise.

\title{
Community intervention in a socially deprived area: nutrition and physical activity; a 10-year follow-up
}

\author{
Pierre Bizel \\ Director of the Education and Health Department, Observatoire de la Santé du Hainaut, Belgium
}

Context: Hainaut (1300000 inhabitants) is a Belgium province highly concerned by socio-economic problems where the health indicators are disadvantageous particularly on obesity. This situation is identified since 1997 by a first health board of Hainaut realised by l'Observatoire de la Santé. (Actual comparison between Hainaut and Europe (I2SARE), Belgium and Walloon Region on middle income, premature mortality and obesity.) Data also show that weight excess and obesity are highly linked to socioeconomic status represented by school level. The lower is the scholar level, the lower is the proportion of people in whom the weight is normal. In Hainaut, almost $60 \%$ living in a family where the highest diploma is a primary school diploma have a weight excess (data close to Belgium and Region Walloon one's). On contrary, in a family where at least one person has a university-level diploma, figures are of $45 \%$ of weight excess (data more disadvantageous than for the country or the region). The social grade is so well present even if it is less marked in Hainaut and this in disfavour of the highest class. The same phenomenon is established for obesity data ( $21 \%$ for the lowest social level and $11 \%$ for the highest).

Program goals: Reduce the social and territorial inequalities of health-by-health promotion actions of proximity on three priority axes: balanced nutrition, regular physical activity and struggle against active and passive tobacco addiction.

Method: A holistic approach of health promotion on the entire provincial territory.

- Place individuals and populations in the centre of the proceeding by information, health education and participation.

- Act on proximity life conditions and on interaction between them (families, districts, communities, schools, etc.) by sensitization, following, sustaining, training, politicians and local professionals linking.

- Contribute to make progress the rules (regulations, law, etc.) and their implementation by communication and coalition, but also to heighten public awareness to health topics and training.

- Contribute to make progress the social representations and pattern by collective communication.

- Follow the whole of the process by developing observation, programming and evaluation tools.

Linking with an institutional-specific network and local institutions on the whole province (social centres, town municipalities, NGO, etc.), the OSH engaged itself in a plural annual actions program.

These actions are connected in different axes:

- Help districts wishing to engage in local health and precariousness programs.

- Create exchange networks for professionals.

- Adapt and relay with professional teams engaged in action the principles messages of health, of prevention of CVD and cancers.

- Create with them specific tools and methods of intervention.

Results: For 10 years, the performing activities to children and young directly or through their life surroundings are followed on both ways, by performing actions evaluation and by weight excess evolution observation and children and young obesity. In 10 years, the evolution of data collected by our inquiries show an increase of overweight (22\% in 1997, 26\% in 2009) and even more obesity, which drop from $5 \%$ to $8 \%$ during the same period. During the 10 years, our strategies and our methods developed in following sectors in a global mean of complexity, of action linking's and partnership reinforcement: the infancy, the school, the youth.

Discussion:

- The struggle against childhood obesity is an integral part of a larger thinking about the place of health promotion and the goal of reduction of social inequalities of health in the public health action. This program 
open more and more on various fields of intervention (nutrition supply, transports, housing, food aid, culture, etc.) notably in link with sustainable development.

- The health education messages receptivity is largely increased by a proximity work and on the long term, but communication work by Medias is still producing effects.

- The need of more precise data on local territories to identify and understand health local inequalities.
- The need of an evaluation system at the level of the stakes complexity and interventions.

- The need of an important and coherent investment at different level of political and social action and from Europe to the district and town. The Region and the Community have tools to promote health in socially deprived territories from a political project of reduction of health inequalities.

\title{
VIASANO: a community-based intervention
}

\author{
Anne Boucquiau \\ Member of the Technical Group of the INAMI on Chronic Diseases and Obesity; Member of BASO (Belgian \\ Association of the Study of Obesity), BSN (Belgian NutritionSociety) and SFN (Societe Francaise de Nutrition)
}

Background: The growing prevalence of childhood obesity throughout Europe increases the need for largescale community-based initiatives aimed at tackling this serious public health concern. Over the last 10 years, studies have demonstrated that the prevention of overweight and obesity is possible through comprehensive and coordinated interventions that support and facilitate physical activity and healthy diets in a variety of settings and through multisectorial engagement. In order to tackle this issue, a long-term school-based nutrition information programme - Fleurbaix Laventie Ville Santé Study (FLVS) - was started in 1992 in two towns in the North of France (Fleurbaix and Laventie) and was followed by a number of community-based interventions over the next 12 years. The results of FLVS study show a decrease in the prevalence of overweight in children (1992: 11.4\% in FLVS and $12 \cdot 6 \%$ in Control towns (CT), $P=0 \cdot 6 ; 2004: 8 \cdot 8 \%$ in FLVS and $17 \cdot 8 \%$ in CT, $P<0 \cdot 0001$ ), observed during the period when the mobilisation of the population became more generalised at community level, involving a wide variety of stakeholders. On the basis of the findings of the FLVS study, the EPODE methodology was developed in 2004 in France, as a coordinated, capacity-building approach for communities to implement effective and sustainable strategies to prevent childhood obesity. Initiated first in ten French pilot towns, EPODE is considered to be an innovative example of a community project aimed at promoting healthy behaviours in children. In 2007, the adaptation of the EPODE methodology in Belgium led to the VIASANO programme.

Method: The VIASANO programme was first launched in two pilot towns as a community-based intervention aimed at preventing childhood obesity, involving local stakeholders in a sustainable way. VIASANO proposes a behaviour-centred approach, with an educational philosophy prompting fun and non-stigmatization of any food and behaviours. It is a positive, concrete and step-by-step learning process on food and physical activity. At national level, a coordination team using social marketing and organizational techniques trains and coaches a local project manager nominated in each VIASANO town by the local authorities. The project manager is provided with tools to mobilize local stakeholders through a local steering committee and local networks. This methodology enables the entire community to be empowered and contribute to create a healthier environment facilitating social norms changes and healthier behaviours. The added value of the methodology is based on critical components such as a strong scientific input, institutional endorsement, evidence-based and social marketing techniques, sustainable resources, brand dynamics and a monitoring and evaluation process.

Results: Today EPODE is implemented in more than 300 towns from six countries: France (EPODE, 226 towns), Belgium (VIASANO, sixteen towns), Spain (THAO, fortytwo towns), Greece (PAIDEIATROFI, thirteen towns), South Australia (OPAL, ten councils) and Mexico (EPODE-5 Pasos, currently launched in pilot municipalities). Evaluation to date is conducted through process and output indicators. First outcome indicators from the French EPODE pilot towns indicate an overall decrease in the prevalence of children overweight including obesity between 2004 and 2009 (from $20 \cdot 6 \%$ to $18 \cdot 8 \%, P<0 \cdot 0001)$. Today, the sixteen VIASANO towns are distributed in all regions and communities, and success to date is measured by a large field mobilization.

Discussion: The EPODE methodology raised a lot of interest across Europe, and to facilitate the implementation of similar community-based interventions in other countries, regions and towns, the EPODE European 